\title{
Genome-wide location analysis reveals a role of TFIIS in RNA polymerase III transcription
}

\author{
Yad Ghavi-Helm, Magali Michaut, Joël Acker, Jean-Christophe Aude, Pierre Thuriaux, \\ Michel Werner, ${ }^{2}$ and Julie Soutourina ${ }^{1}$ \\ CEA, iBiTec-S, Gif-sur-Yvette Cedex F-91191, France
}

\begin{abstract}
TFIIS is a transcription elongation factor that stimulates transcript cleavage activity of arrested RNA polymerase II (Pol II). Recent studies revealed that TFIIS has also a role in Pol II transcription initiation. To improve our understanding of TFIIS function in vivo, we performed genome-wide location analysis of this factor. Under normal growth conditions, TFIIS was detected on Pol II-transcribed genes, and TFIIS occupancy was well correlated with that of Pol II, indicating that TFIIS recruitment is not restricted to NTP-depleted cells. Unexpectedly, TFIIS was also detected on almost all Pol III-transcribed genes. TFIIS and Pol III occupancies correlated well genome-wide on this novel class of targets. In vivo, some dst1 mutants were partly defective in tRNA synthesis and showed a reduced Pol III occupancy at the restrictive temperature. In vitro transcription assays suggested that TFIIS may affect Pol III start site selection. These data provide strong in vivo and in vitro evidence in favor of a role of TFIIS as a general Pol III transcription factor.
\end{abstract}

[Keywords: TFIIS; transcription; RNA polymerase III; RNA polymerase II; ChIP-chip]

Supplemental material is available at http://www.genesdev.org.

Received January 21, 2008; revised version accepted May 23, 2008.

Gene transcription is a complex and highly regulated process that, in eukaryotes, is carried out by three specialized RNA polymerases (Pol I, II, and III), dedicated to the transcription of different sets of genes. Transcription starts by the assembly of large preinitiation complexes (PIC) comprising TBP (the TATA-binding protein common to all three transcription systems) and general transcription factors specific to the RNA polymerase considered. Other transcription factors facilitate RNA elongation by Pol II (Shilatifard et al. 2003). One of these elongation factors, TFIIS, was initially discovered in human (Natori et al. 1973) and yeast cells (Sawadogo et al. 1980b), and is highly conserved in the eukaryotic and archaeal kingdoms (Hausner et al. 2000; Fish and Kane 2002). Structurally unrelated but functionally equivalent factors (GreA and GreB) also operate in bacteria (Borukhov et al. 1993; Opalka et al. 2003). TFIIS forms a binary complex with Pol II and stimulates an intrinsic transcript cleavage activity of RNA polymerase allowing elongating enzymes to resume RNA synthesis after accidental transcription arrest (Fish and Kane 2002; Kettenberger et al. 2003).

TFIIS is organized in three domains as determined by

Corresponding authors

${ }^{1}$ E-MAIL julie.soutourina@cea.fr; FAX 33-1-69-08-47-12.

2E-MAIL michel.werner@cea.fr; FAX 33-1-69-08-47-12.

Article is online at http://www.genesdev.org/cgi/doi/10.1101/gad.471908. limited proteolysis and nuclear magnetic resonance (NMR) (Awrey et al. 1997; Olmsted et al. 1998). The three-dimensional structure of the Pol II-TFIIS complex, without the TFIIS N-terminal domain, has been determined by crystallographic studies (Kettenberger et al. 2003). The Nterminal domain I of TFIIS protrudes from Pol II and is not required for elongation (Nakanishi et al. 1995; Awrey et al. 1998), but binds the yeast Mediator and SAGA coactivator (Wery et al. 2004). The TFIIS central domain II is inserted into the funnel-shaped pore of Pol II and brings the C-terminal conserved RSADE motif very close to the enzyme active site (Kettenberger et al. 2003). Remarkably, the C-terminal domain III of TFIIS shares the RSADE motif with the C-terminal parts of the Rpa12 (Pol I) and Rpc11 (Pol III) subunits, and is more distantly related to the corresponding Rpb9 subunit of Pol II, which has no RSADE motif. Rpc11 mediates the intrinsic transcription cleavage activity of Pol III (Chedin et al. 1998).

In the yeast Saccharomyces cerevisiae, TFIIS is encoded by DST1. The dst1- $\Delta$ mutant, or $d s t 1$ mutants of the RSADE motif, grow like wild type, but are sensitive to the NTP-depleting effects of 6-azauracil (6AU) and mycophenolic acid (MPA), two drugs thought to compromise elongation efficiency (Exinger and Lacroute 1992; Ubukata et al. 2003). In the presence of 6-azauracil, deletion of DST1 affects Pol II processivity without influencing its elongation rate (Mason and Struhl 2005). TFIIS was also 
found to stimulate transcription past an artificial arrest site in vivo, thus further supporting the notion that it overcomes elongation blocks (Kulish and Struhl 2001). Recent reports suggest that, in addition to its well-documented role as a Pol II elongation factor, TFIIS also contributes to transcription initiation (Malagon et al. 2004; Wery et al. 2004; Prather et al. 2005; Guglielmi et al. 2007; Kim et al. 2007). This initiation role does not depend on the C-terminal RSADE motif needed for transcript cleavage stimulatory activity (Guglielmi et al. 2007; Kim et al. 2007).

Although the role of TFIIS in stimulating Pol II transcript cleavage activity is well characterized in vitro, its in vivo function remains less obvious. In this study, we analyzed the genome-wide location of TFIIS using chromatin immunoprecipitation coupled with DNA microarray hybridization (ChIP-chip). TFIIS was detected on many Pol II-transcribed genes under normal growth conditions, and its occupancy closely correlated with that of Pol II. Unexpectedly, we identified a novel class of TFIIS targets corresponding to Pol III-transcribed genes. In vitro Pol III transcription assays suggested that TFIIS may contribute to correct start site selection. Taken together, our in vivo and in vitro data reveal a previously unsuspected role of TFIIS in Pol III transcription.

\section{Results}

TFIIS is located genome-wide on the Pol II- and Pol III-transcribed genes

Previous models proposed that TFIIS protein was recruited during transcription elongation in conditions where Pol II was stalled. This hypothesis was strengthened by ChIP assays, suggesting that TFIIS does not normally reside on DNA but is specifically recruited in the presence of NTP-depleting drugs that favor the transcriptional arrest of Pol II elongation complexes (Pokholok et al. 2002). However, recent ChIP analyses revealed the presence of TFIIS on promoters and coding regions of several selected genes in cells grown under normal conditions (Prather et al. 2005; Guglielmi et al. 2007). To better understand TFIIS function and resolve this discrepancy, we performed genome-wide location analysis of TFIIS under standard conditions, or in cells that have been NTP-depleted in the presence of MPA.

We examined the genome-wide distribution of TFIIS and Pol II by ChIP-chip experiments and compared their relative enrichments. The DNA arrays used contained $>40,000$ oligonucleotide probes covering $12 \mathrm{Mb}$ of the yeast genome (see the Materials and Methods). This analysis was done using a strain (YGH2) carrying an active $\mathrm{N}$ terminal HA-tagged version of TFIIS. We found 3652 oligonucleotides significantly bound $(P$-value $<0.005)$ by TFIIS under standard conditions. Correlation between TFIIS and Pol II enrichment showed an unexpected pattern (Fig. 1A). The distribution was split into two highly different data sets. A first set of oligonucleotides, located within 1419 Pol II-transcribed genes, showed a good correlation between TFIIS and Pol II enrichment. As dis- cussed below, a second group of oligonucleotides was enriched for TFIIS but not for Pol II, and corresponded to Pol III-transcribed genes.

The results obtained by ChIP-chip experiments were confirmed by conventional ChIP on a set of selected Pol II- and Pol III-transcribed genes (Fig. 1B). All selected genes displayed a significant enrichment compared to background level measured on the coding region of the nontranscribed GAL1 gene. Similar results were obtained in ChIP experiments using chromatin from a strain expressing native TFIIS and anti-TFIIS antibodies for immunoprecipitation (data not shown). We wondered whether TFIIS could also be detected on Pol I-transcribed 35S rDNA and Pol III-transcribed 5S rDNA templates. Since the array used had a poor coverage of that region, we performed conventional ChIP experiments on the rDNA locus. A slight enrichment on the 35S rDNA and no significant enrichment on the 5S rDNA were seen compared to the intergenic NTS1 and NTS2 regions (Fig. 1C). Since the binding of TFIIS to transcribed sequences would also be observed if TFIIS was an RNA-binding protein, we tested if the enrichment of TFIIS was dependent on the presence of the transcribed RNA by digestion with RNase A/T1 in ChIP experiments. Digestion did not affect TFIIS enrichment (data not shown). We also examined TFIIS occupancy of a gene transcribed by a heterologous T7 RNA polymerase (Chen et al. 1987). We did not observe any significant TFIIS enrichment (Supplemental Fig. S1), indicating that TFIIS does not localize to transcribed sequences thanks to an RNAbinding property.

\section{Genome-wide TFIIS occupancy on Pol II-transcribed genes correlates with Pol II occupancy}

Analysis of Pol II genome-wide occupancy revealed 15,911 oligonucleotides significantly bound ( $P$-value $<0.005)$. These probes corresponded to 3819 Pol II-transcribed genes. After removing all the oligonucleotides corresponding to Pol III-transcribed genes from the analysis, there was a fairly linear relationship between TFIIS and Pol II enrichment with a correlation coefficient of 0.642 (Fig. 1A). The example of Pol II and TFIIS enrichment profiles on the PYK1 gene (Fig. 1D) confirms that TFIIS is present on coding regions even under standard growth conditions (i.e., in the absence of NTP-depleting drugs), and that TFIIS and Pol II occupancies are extremely well correlated. Other examples of enrichment profiles on $A D H 1$ and PGK1 genes are shown in Supplemental Figure S2A,B. The presence of TFIIS is not limited to transcribed protein-coding regions, since we detected TFIIS on intergenic regions transcribed in short unstable RNAs. The examples of the cryptic transcript NELO25C, located on chromosome $\mathrm{V}$ between RMD6 and DLD3 genes (Wyers et al. 2005), and of SRG1, a transcriptional repressor of SER3 (Martens et al. 2004), are shown in Supplemental Figure S2C,D. Pol II- and TFIIS-enriched genes were classified according to the biological process categories of gene ontology (GO). A standard hypergeometric test was used to determine the overrepresented 
A
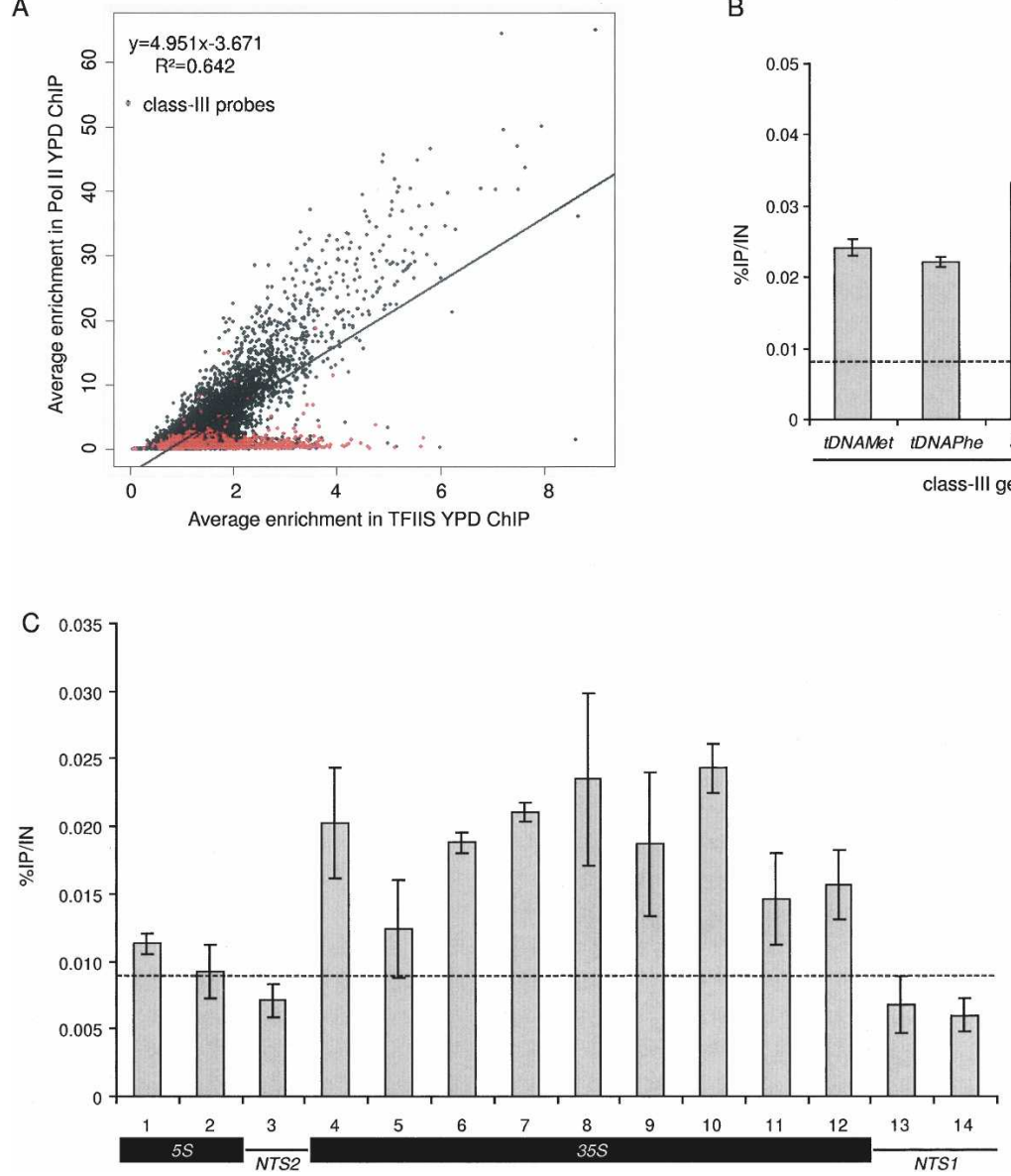

B

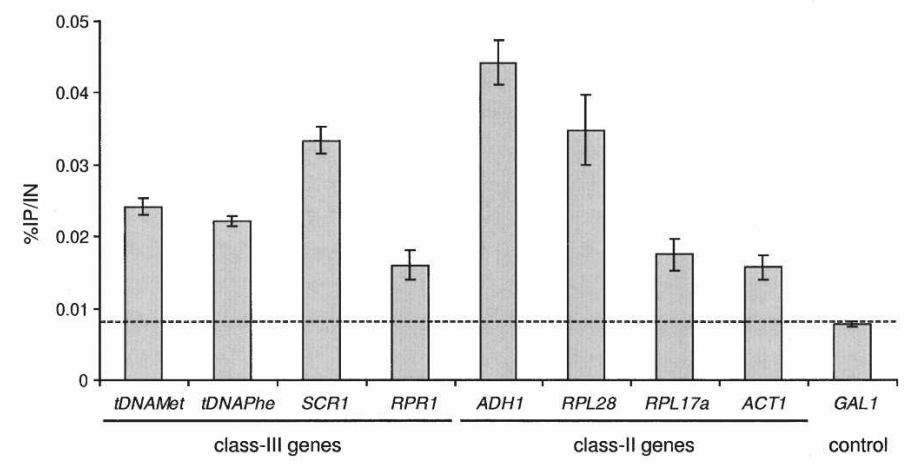

Figure 1. Genome-wide location analysis of TFIIS and Pol II. (A) Enrichment of Pol II versus enrichment of TFIIS. The genome-wide enrichment of Pol II and TFIIS in YPD medium was assessed from ChIP-chip experiments using YGH2 (3HA-TFIIS) strain. A linear regression for Pol II enrichment versus TFIIS enrichment on class II probes and its equation are indicated. Red dots correspond to class III probes. (B) Quantitative ChIP analysis of TFIS enrichment on selected genes. Immunoprecipitated fragments from ChIP experiments were amplified with primers as indicated in the Supplemental Material. The GAL1 ORF was used as a control. Error bars represent the standard deviation between at least three biological replicates. The background level was represented by a dotted line. (C) Quantitative ChIP analysis of TFIIS enrichment on rDNA. Immunoprecipitated fragments from ChIP experiments were amplified with primers as indicated in the Supplemental Material. The intergenic NTS1 and NTS2 regions were used as a control. Error bars represent the standard deviation between at least three biological replicates. The background level was represented by a dotted line. (D) Enrichment profile of Pol II and TFIIS on the PYK1 gene. The enrichment of Pol II (blue) and TFIIS (red) on PYK1 in YPD medium was assessed from ChIP-chip experiments using YGH2 (3HA-TFIIS) strain. The genomic positions of probe regions on chromosome 1 are indicated along the $X$-axis and represented by black points or circles. Watson strand-transcribed gene PYK1 is colored in blue. The enrichment ratio is indicated along the $Y$-axis.

categories and associated $P$-values. Since the lists of Pol II and TFIIS overrepresented GO categories were largely overlapping (Supplemental Table S1), we concluded that TFIIS was not preferentially bound to a particular group of Pol II-transcribed genes. The smaller number of TFIISenriched genes compared to Pol II is best explained by the lower binding levels of TFIIS.

To explore the effect of NTP depletion on TFIIS genome-wide occupancy, yeast cells grown in minimal SD medium were exposed to $10 \mu \mathrm{g} / \mathrm{mL}$ MPA for $4 \mathrm{~h}$ (i.e., just when an effect on growth rate can be observed). We could detect a slight increase of TFIIS enrichment level compared to standard conditions (SD medium), but this effect was far less pronounced than in a previous study using a different and nonfunctional tagged version of TFIIS (Supplemental Fig. S3A; Pokholok et al. 2002). The enrichment profiles of TFIIS on PYK1 and ILV5 genes illustrate that in the presence of mycophenolate, occupancy levels of TFIIS were only slightly increased all along the genes (Supplemental Fig. S3B,C). Note that TFIIS enrichment is much higher in yeast cells growing rapidly in rich medium as compared with minimal medium.

\section{Genome-wide TFIIS occupancy on Pol III-transcribed genes correlates with Pol III occupancy}

We compared TFIIS and Pol III occupancies on Pol IIItranscribed genes, since a global correlation would imply 
that TFIIS might be a new Pol III general factor. A ChIPchip experiment on Rpc160, the largest Pol III subunit, was performed. We found 964 enriched oligonucleotides located in or close to the class III genes that were described previously in genome-wide location analyses (Harismendy et al. 2003; Roberts et al. 2003; Moqtaderi and Struhl 2004). Four-hunderd-thirty-two of the 3652 oligonucleotides significantly bound $(P$-value $<0.005)$ by TFIIS under standard conditions were within or close (up to $500 \mathrm{bp}$ upstream or downstream) to class III genes. They were significantly enriched by Pol III, and represented all Pol III-transcribed genes for which probes were located on the arrays; i.e., $t D N A s, S C R 1, R P R 1, S N R 52$, and ZOD1. SNR6 showed a low level of TFIIS enrichment on the B box located $\sim 100$ bp downstream from transcribed region (Supplemental Fig. S4A). No TFIIS enrichment was detected on the ETC loci that are occupied by TFIIIC but not by TFIIIB and Pol III (Moqtaderi and Struhl 2004). As noted above, the 5S rDNA also showed no detectable enrichment for TFIIS.

The overall correlation between Pol III and TFIIS occupancies (Fig. 2A) confirmed our previous observations on the genome-wide TFIIS distribution. Considering only the oligonucleotides corresponding to Pol III-transcribed genes, the analysis showed a linear relationship with a correlation coefficient of 0.718 (Fig. 2A). The enrichment profile of TFIIS on SCR1, a selected class III gene, is presented in Figure 2B. Other examples of TFIIS enrichment profiles on RPR1 and $t D N A^{G l y}$ are shown in Supplemental Figure S4B,C. In these examples, the Pol II-transcribed genes adjacent to class III genes were devoid of TFIIS, strongly suggesting that TFIIS could be recruited independently of Pol II transcription.

To establish that the presence of TFIIS on class III genes was specific to Pol III transcription and independent of Pol II transcription, we used specific Pol II and

A

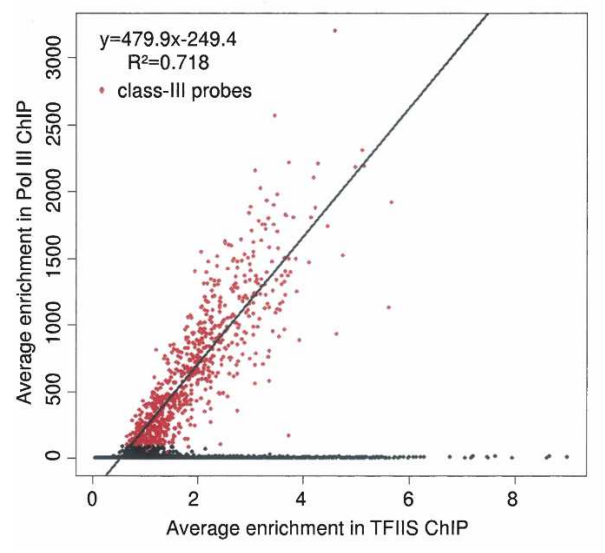

Pol III mutations and examined how they affected the distribution of TFIIS. The rpb1-1 mutant of Pol II rapidly stops transcription after a shift to $37^{\circ} \mathrm{C}$ (Nonet et al. 1987). When cells were incubated at $37^{\circ} \mathrm{C}$ for $30 \mathrm{~min}$, Pol II occupancy of all Pol II-transcribed genes tested $(A D H 1$, RPL28, RPL17a, and ACT1) was greatly reduced in the rpb1-1 strain compared to the wild-type strain (Fig. 3A, left panel). TFIIS occupancy strongly correlated with that of Pol II, and was also greatly reduced after incubation at $37^{\circ} \mathrm{C}$. Conversely, TFIIS and Pol III occupancies of all Pol III-transcribed genes tested (tDNAMet, tDNAPhe, SCR1, and $R P R 1$ ) remained largely unchanged under the same conditions (Fig. 3A, right panels). These results demonstrated that the presence of TFIIS on Pol III-transcribed genes is independent of Pol II transcription.

The Pol III-specific mutant rpc25-S100P was similarly used to impair class III gene transcription. For this purpose, a more prolonged shift to the restrictive temperature $\left(10 \mathrm{~h}\right.$ at $\left.37^{\circ} \mathrm{C}\right)$ was needed (Zaros and Thuriaux 2005). Under this condition, Pol III and TFIIS occupancies were reduced on class III genes, even though the decrease of TFIIS occupancy was somewhat less pronounced (Fig. 3B, right panel). As expected, no significant effect was observed on Pol II and TFIIS association to the Pol IItranscribed genes (Fig. 3B, left panel). Thus, the presence of TFIIS on class II or III genes is largely dependent on transcription by Pol II or Pol III, respectively.

Most class III genes are too small to allow a spatial resolution of the Pol III machinery location by ChIP, but SCR1, the longest Pol III-transcribed gene (522 bp), provided this possibility. We examined the spatial distribution of TFIIS on this gene compared with that of the Pol III basal machinery composed, in addition to Pol III, of TFIIIB and TFIIIC complexes (Geiduschek and Kassavetis 2001). Seven real-time PCR amplicons spanning the upstream, transcribed, and downstream regions of

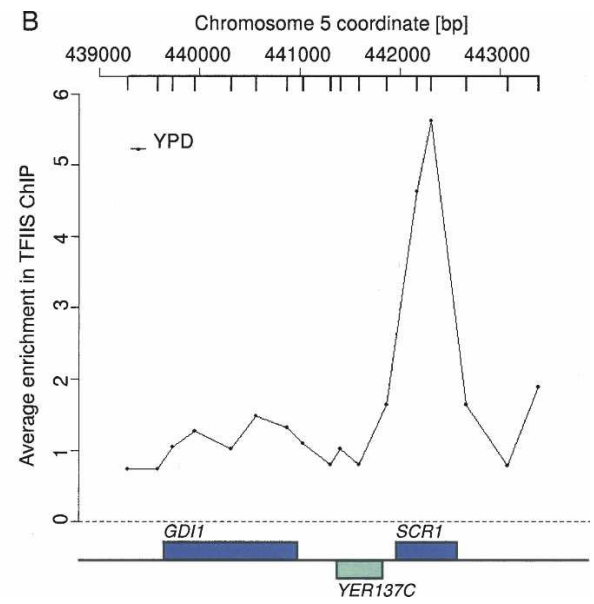

Figure 2. Genome-wide location analysis of TFIIS and Pol III. (A) Enrichment of Pol III versus enrichment of TFIIS. The genome-wide enrichment of Pol III and TFIIS in YPD medium was assessed from ChIP-chip experiments with MW671 (3HA-RPC160) and YGH2 (3HA-TFIIS) strains, respectively. A linear regression and its equation are indicated. Red dots correspond to class III probes. (B) Enrichment profile of TFIIS on the SCR1 gene. The genome-wide enrichment of TFIS in YPD medium was assessed from ChIP-chip experiments. The genomic positions of probe regions on chromosome 5 are reported along the $X$-axis and represented by black points. Watson strand-transcribed genes are colored in blue and Crick strand-transcribed genes are colored in green. The enrichment ratio is reported along the $Y$-axis. 
Ghavi-Helm et al.
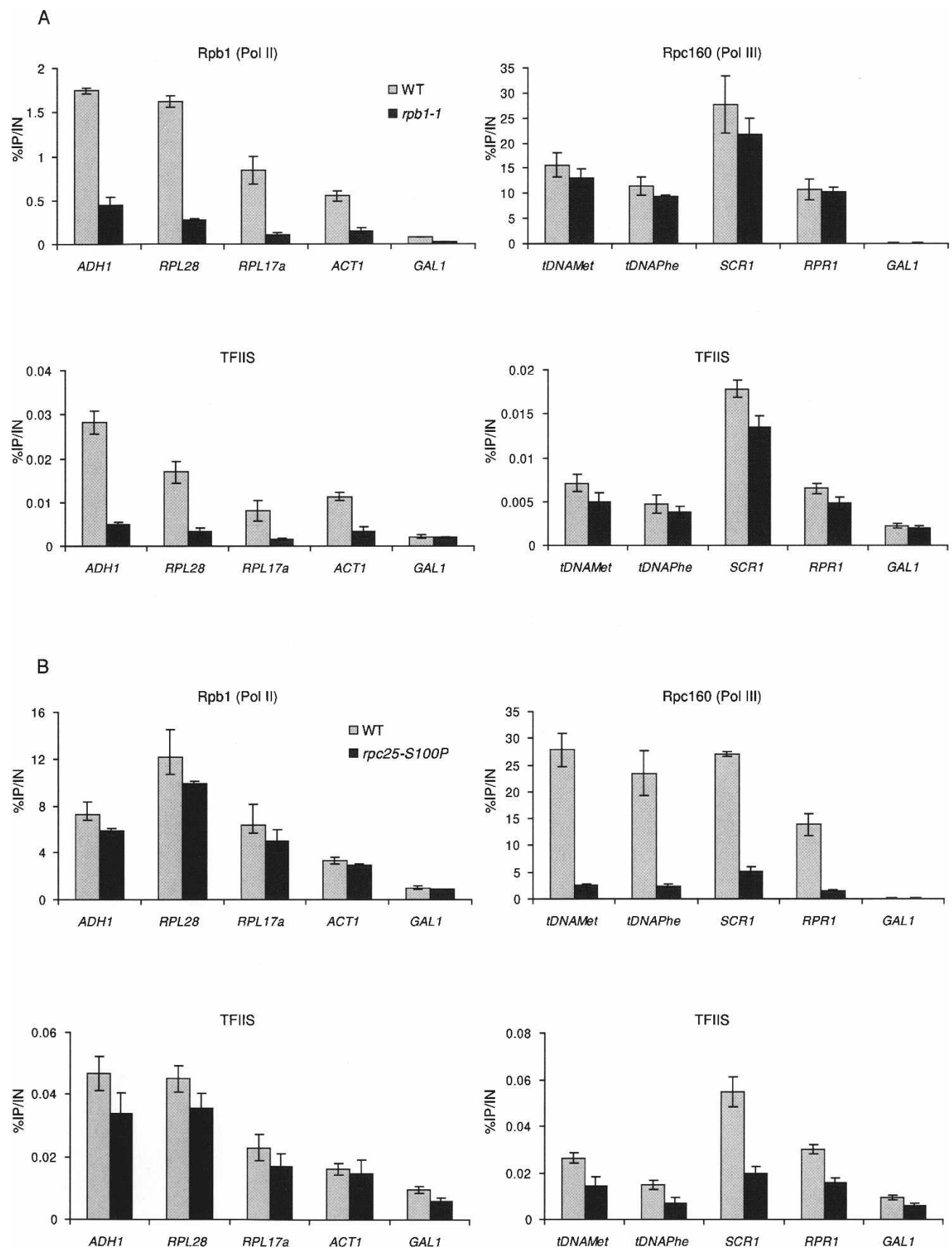

Figure 3. Effect of $r p b 1-1$ and $r p c 25-S 100 P$ mutations on TFIIS, Pol II, and Pol III occupancies on selected genes. Immunoprecipitations were performed using antibodies against 3HA (12CA5) for HA-TFIIS, 13Myc (9E10) for Rpc160-13Myc, and CTD (8WG16) for the Pol II Rpbl subunit. Immunoprecipitated fragments from ChIP experiments were amplified with primers as indicated in the Supplemental Material. The GAL1 ORF was used as a control. The values are the average of three independent experiments. Error bars indicate the standard deviation. (A) TFIIS, Pol II, and Pol III occupancies in the rpb1-1 mutant. Standard ChIP assays were performed on chromatin prepared from the D788-4a strain transformed by RPB1-containing pYeB-B220 plasmid (wild type) or by empty vector Yep351 (rpb1-1). Cells were grown in selective SD medium complemented with amino acids at $30^{\circ} \mathrm{C}$ and then shifted for 30 min at $37^{\circ} \mathrm{C}$. (B) TFIIS, Pol II, and Pol III occupancies in the rpc25-S100P mutant. Standard ChIP assays were performed on chromatin prepared from D792-3a strain transformed by RPC25-containing pRS315-RPC25 plasmid (wild type) or by empty vector pRS315 (rpc25-S100P). Cells were grown in selective SD medium complemented with amino acids at $30^{\circ} \mathrm{C}$ and then shifted for $10 \mathrm{~h}$ at $37^{\circ} \mathrm{C}$.

SCR1 were designed (Fig. 4A). We analyzed the association profiles of the Rpc160 Pol III subunit, the Bdp1 TFIIIB subunit, the Tfe1 TFIIIC subunit, and TFIIS (Fig.
4B). The distribution of the Pol III transcription machinery on SCR1 was as described previously (Roberts et al. 2006). TFIIIB binding was maximal on the TATA box, 
A

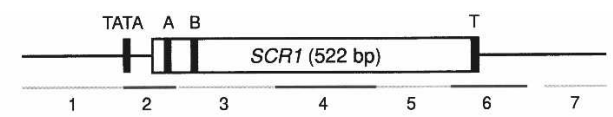

B
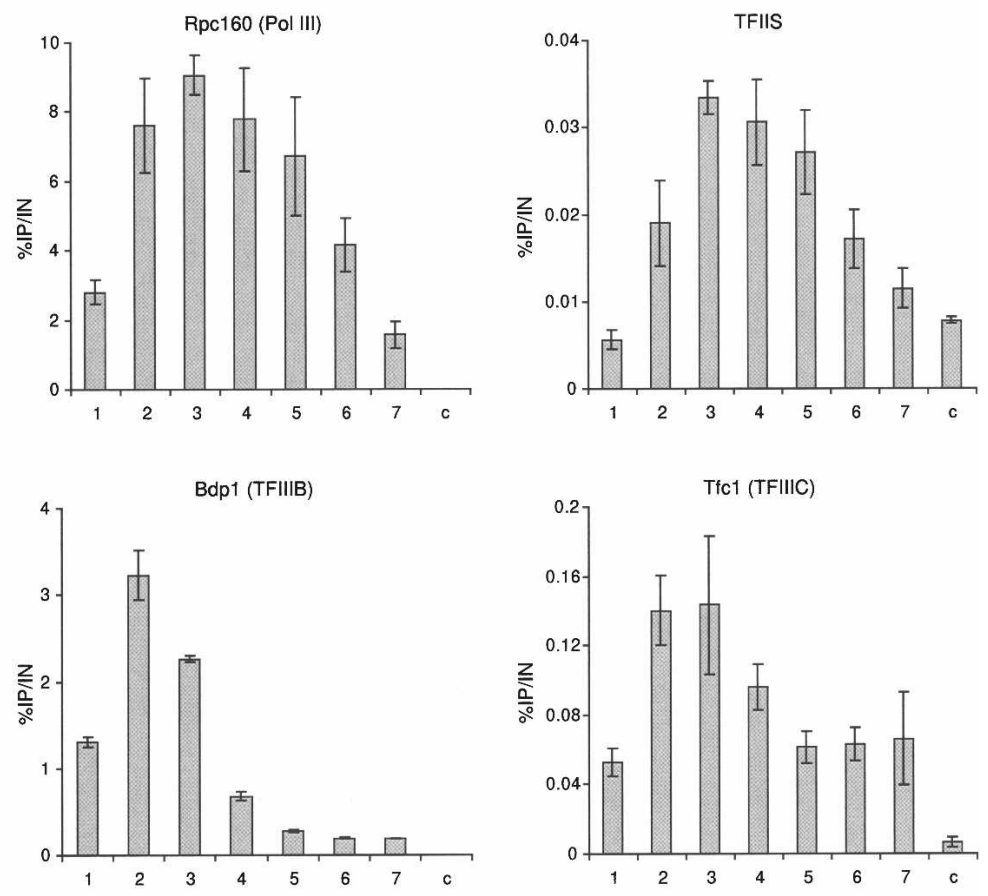

Pol III, TFIIS, TFIIIB, and TFIIIC location analysis on the SCR1 gene. (A) Schematic organization of the SCR1 gene. The location of the PCR fragments amplified in ChIP analyses are indicated by gray and black lines. Black boxes represent TATA, A and $\mathrm{B}$ boxes, and Terminator $(\mathrm{T})$. $(B)$ Occupancy profile of Pol III, TFIIS, TFIIIB, and TFIIIC on the SCR1 gene. Standard ChIP assays were performed on chromatin prepared from YGH11 strain using antibodies against 3HA-TFIIS (12CA5) and Rpc160-13Myc (9E10), from MW4035 strain using antibodies against Bdp1-3HA (12CA5), from yOH1 strain using antibodies against Tfc1-13Myc (9E10), and from YGH15 strain using antibodies against Rpc11-3HA (12CA5). Cells were grown in YPD medium at $30^{\circ} \mathrm{C}$. The GAL1 ORF was used as a control (c). while TFIIIC was cross-linked over the entire SCR1 locus. TFIIS distribution on the SCR1 gene resembled more closely that of Pol III with a maximum at the level of the A and $\mathrm{B}$ boxes at the beginning of the gene. However, the TFIIS occupancy profile differed from those of TFIIIC and TFIIIB. We also examined the association of Rpc11, a Pol III subunit homologous to TFIIS, and showed that the distribution of Rpc11 on the SCR1 gene was similar to that of Rpc160 subunit (Fig. 4B). Thus, the TFIIS distribution on the SCR1 gene follows closely that of Pol III, suggesting an active role of TFIIS in class III-gene transcription.

Since TFIIS interacts directly with Pol II, we tested the possible association of this factor with Pol III machinery by coimmunoprecipitation (co-IP) approach. No interaction of TFIIS with Pol III, TFIIIC, and TFIIIB was detected in crude extracts from noncross-linked cells (Supplemental Fig. S5A). However, when co-IP assays were performed with extracts from cross-linked cells (ChIP extracts), TFIIS coimmunoprecipitated with Pol III and TFIIIC, but not with TFIIIB (Supplemental Fig. S5B), in agreement with our ChIP data. We detected TFIIS when Rpc160-Myc or Rpc11-HA were used to immunoprecipitate Pol III. These co-IP results showed a concomitant presence of TFIIS with Pol III and TFIIIC on class III genes.

Effect of dst1 mutations on tRNA synthesis and Pol III occupancy in vivo

Since tRNA suppressor genes are transcribed by Pol III, we tested the influence of deleting DST1 on suppression efficiency to analyze the role of TFIIS in Pol III transcription in vivo. We therefore compared the growth of a yeast strain containing the chromosomally integrated SUP11 tRNA $^{\text {Tyr }}{ }_{\text {UAA }}$ allele suppressing the ade2-1 ochre mutation with that of the isogenic dst1- $\Delta$ strain. In the presence of adenine in the growth medium, the SUP11 
dst1- $\Delta$ ade2-1 strain grew as well as the SUP11 DST1 ade2-1 strain (Fig. 5A, +Ade), but a significant growth difference was observed between the two strains in the medium lacking adenine (-Ade), indicating a decrease in the level of ade2-1 mutation suppression. This observation suggests that TFIIS could influence Pol III transcription in vivo.

In the course of this study, we observed that dst1- $\Delta$ impaired yeast growth at $16^{\circ} \mathrm{C}$ (Fig. 5B), a phenotype that has not been described previously. This phenotype was not complemented by a point mutation in the RSADE motif of TFIIS domain III, responsible for the cleavage activity (dst1-E291A) (Fig. 5C). This mutant, the most sensitive to cold temperature, actually had a growth defect even at $30^{\circ} \mathrm{C}$. In contrast, the $d s t 1-R 200 A$ mutant in Pol II-binding domain II grew like a wild type. We con- cluded that the domain III of TFIIS is important for yeast growth at low temperature.

We analyzed the effect of dst1 deletion on the levels of short-lived $\mathrm{tRNA}_{3}{ }^{\mathrm{Leu}}$ precursor at low temperature. Wild-type strain and a $d s t 1-\Delta$ mutant were grown at $30^{\circ} \mathrm{C}$ or shifted for $8 \mathrm{~h}$ at $16^{\circ} \mathrm{C}$. Total RNAs were extracted and used for Northern blot analysis (Fig. 5D). The amount of mature tRNA ${ }_{3}^{\text {Leu }}$ was used as a loading control. The deletion of dst1 significantly decreased the tRNA $_{3}{ }^{\text {Leu }}$ precursor levels in yeast cells grown at $16^{\circ} \mathrm{C}$ (2.1-fold).

To investigate the effect of $d s t 1$ mutations on class III gene expression under low-temperature conditions $\left(16^{\circ} \mathrm{C}\right)$ in more details, we performed a genome-wide analysis of Pol III transcriptome (Ciesla et al. 2007). $\log _{2}$ ratios of class III gene transcription levels in the mutant versus
Figure 5. In vivo effect of TFIIS on Pol III transcription. (A) Growth of the YGH10 (SUP11 dst1- $\Delta$ ade2-1 ochre) strain relative to the MB159-2c (SUP11 DST1 ade2-1 ochre) strain. Cells were grown at $30^{\circ} \mathrm{C}$ for $3 \mathrm{~d}$ on minimal medium supplemented (+Ade) or not (-Ade) with adenine. (B) Growth of the dst1-null mutant at $30^{\circ} \mathrm{C}$ or $16^{\circ} \mathrm{C}$. Suspensions of YPH500 (wildtype) or ESH1 (dst1- $\Delta)$ strains were serially diluted, spotted on YPD-rich medium, and grown for $3 \mathrm{~d}$ at $30^{\circ} \mathrm{C}$ or at for $5 \mathrm{~d} 16^{\circ} \mathrm{C}$. (C) Growth of $d s t 1$ mutants at $30^{\circ} \mathrm{C}$ or $16^{\circ} \mathrm{C}$. dst1- $\Delta$ (YGH12) strain was transformed by pRS425-pPGK vector, pRS425DST1, pRS425-dst1R200A, or pRS425dst1E291A plasmids bearing different DST1 alleles. Cells were grown in liquid SC-leucine medium at $30^{\circ} \mathrm{C}$ or shifted at time 0 at $16^{\circ} \mathrm{C} .(D)$ Northern blot analysis of tRNA $_{3}{ }^{\mathrm{Leu}}$ abundance in wild-type and dst1- $\Delta$ strains. RNAs were extracted from wild-type (ESH1/pRS425-DST1) and dst1- $\Delta$ (ESH1/pRS425-pPGK) cells grown in SCleucine medium at $30^{\circ} \mathrm{C}$ or shifted at $16^{\circ} \mathrm{C}$ for $8 \mathrm{~h}$. tRNA precursors abundance was quantified, and fold decrease of transcription in dst1- $\Delta$ strain was compared with that in wild type grown at the same temperature (arbitrary set to 1). Average values of three independent experiments and standard deviations (SD) are indicated. (E) Analysis of the Pol III transcriptome at $16^{\circ} \mathrm{C}$. RNAs were extracted from the dst1- $\Delta$ (ESH1) strain transformed by pRS425-pPGK vector, pRS425-DST1, pRS425-dst1R200A, or pRS425-dst1E291A plasmids grown at $30^{\circ} \mathrm{C}$ or shifted for $8 \mathrm{~h}$ to $16^{\circ} \mathrm{C}$, and analyzed by hybridization to Pol III-specific microarrays. Expression $\log _{2}$ ratios of class III genes in the mutant strains compared with wild type are presented according to the red-green color scale.
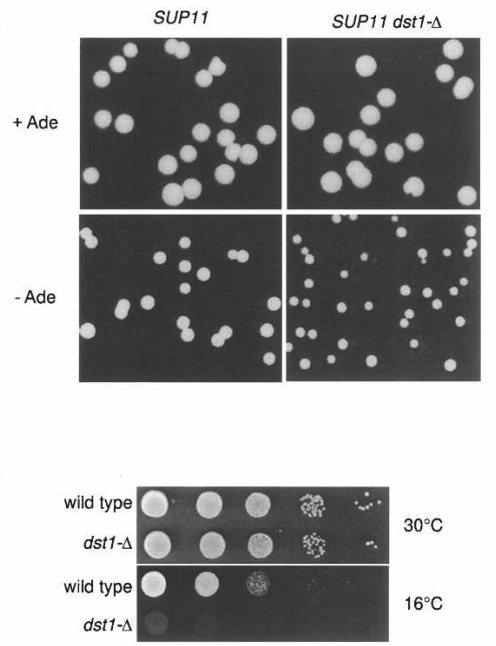

C
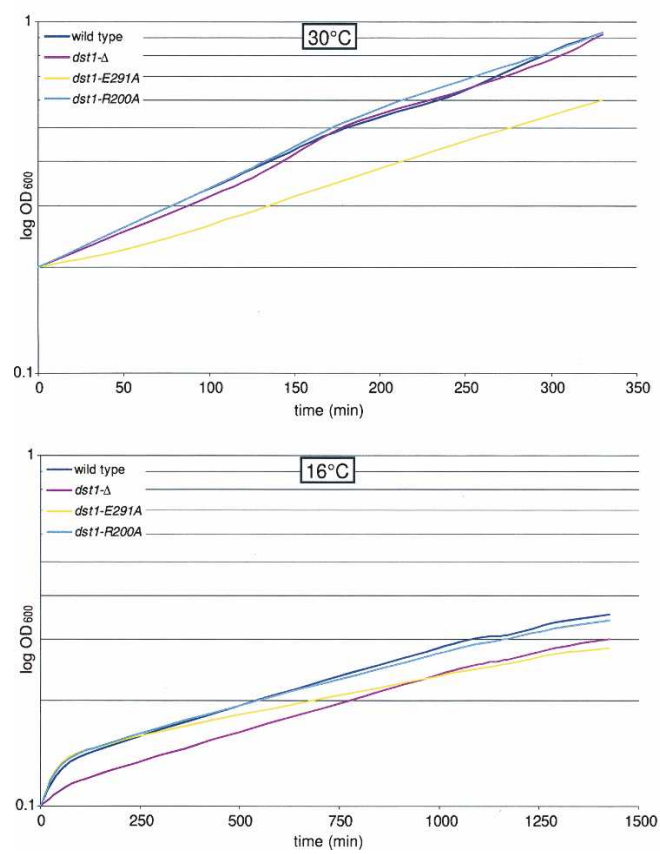

D

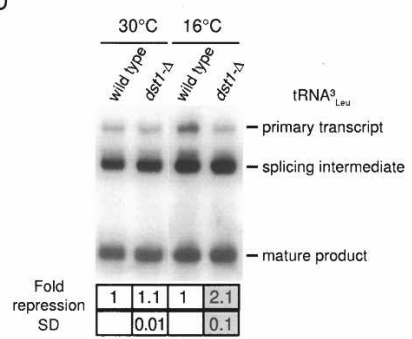

E

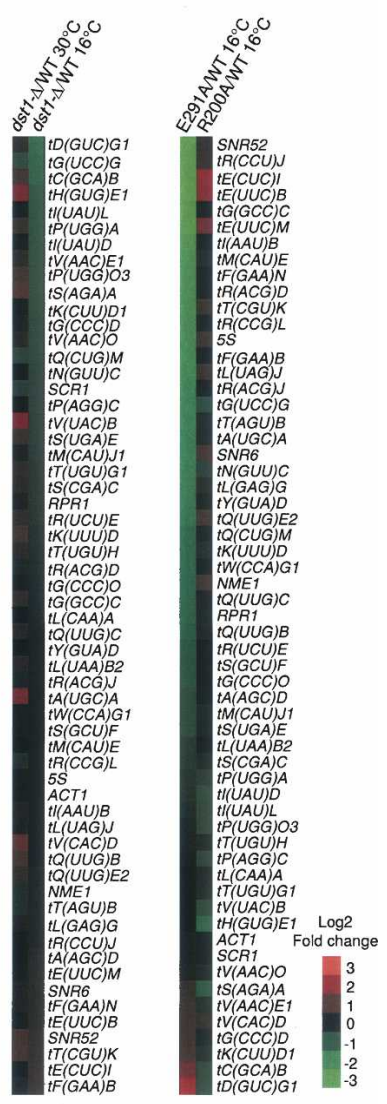


wild-type strains were hierarchically clustered (Fig. 5E). At $30^{\circ} \mathrm{C}$, the Pol III transcript profiles of the $d s t 1-\Delta$ and the wild-type strains were similar. In contrast, a significant decrease of several class III transcripts was observed in $d s t 1-\Delta$ mutant at $16^{\circ} \mathrm{C}$. We found that transcription levels of class III genes were strongly diminished at $16^{\circ} \mathrm{C}$ in the dst1-E291A strain, whereas the dst1-R200A mutant had a Pol III transcript profile similar to that of the wild type. The dst1-E291A mutant had more pronounced effects on Pol III transcription than the dst1- $\Delta$ strain, consistent with its slower growth at all temperatures. Curiously, class III genes showing the most reduced transcript levels were different in dst1- $\Delta$ and dst1E291A mutants.

To examine the effect of the $d s t 1-\Delta$ mutation on genome-wide Pol III occupancy at low temperature $\left(16^{\circ} \mathrm{C}\right)$, wild-type and dst1- $\Delta$ strains containing a C-terminal $13 \mathrm{Myc}$ tag on the Rpc160 Pol III subunit were grown in YPD-rich medium at $30^{\circ} \mathrm{C}$ and then shifted to $16^{\circ} \mathrm{C}$ for 8 h. At low temperature, all class III genes were bound by Pol III. We compared Pol III genome-wide occupancy in a $d s t 1-\Delta$ and a wild-type strain grown at $16^{\circ} \mathrm{C}$ (Fig. 6A). A general reduction of Pol III occupancy in the dst1- $\Delta$ mutant was observed, suggesting that TFIIS could stabilize Pol III on class III genes. Regression analysis indicated a 1.5-fold decrease of Pol III binding in the mutant (the slope of the correlation line, shown in red, equal to $0.645)$ with a high correlation coefficient $\left(R^{2}=0.965\right)$. The high correlation coefficient indicated that the association of Pol III to almost all class III genes was significantly reduced. A similar analysis was performed to examine the effect of $d s t 1$ deletion on Pol II genome-wide occupancy at low temperature (Fig. 6B). The binding of Pol II was found to be reduced 1.6-fold in the mutant (the slope of the correlation line equal to 0.621 ) with a reduced correlation between wild-type and $d s t 1-\Delta$ strains $\left(R^{2}=0.647\right)$. In the case of Pol II, the $d s t 1$ deletion could have indirect effects on gene expression regulation that may explain the lower correlation coefficient between the wild-type and the mutant strains. Examples of enrichment profiles on specific genes are shown in Supplemental Figure S6. The overall reduction of Pol II and Pol III occupancies that was observed at low temperature when dst1 was deleted suggested that TFIIS could stabilize Pol II and Pol III on their target genes.

We extended our genome-wide Pol III and Pol II location analysis to the dst1-E291A and $d s t 1-R 200 A$ point mutants grown at $16^{\circ} \mathrm{C}$. The binding of Pol II was found to be reduced 1.4-fold in the dst1-E291A mutant and 1.3 -fold in the dst1-R200A mutant (the slope of the correlation lines equal to 0.723 and 0.788 , respectively) (Fig. $6 \mathrm{D}, \mathrm{F})$. As expected, both dst1 mutations affected Pol II occupancy. In contrast, the binding of Pol III was significantly diminished (3.1-fold) in the dst1-E291A mutant, but not at all in the $d s t 1-R 200 A$ mutant (the slope of the correlation lines equal to 0.325 and 1.088 , respectively) (Fig. 6C,E). Thus, the dst1-E291A mutant affected both Pol II and Pol III binding, whereas the dst1-R200A mutant had a small but significant effect only on Pol II association.
We further analyzed the occupancy of the Bdp1 TFIIIB subunit and the Tfe1 TFIIIC subunit in dst1- $\Delta$ and wildtype strains on several class III genes at $16^{\circ} \mathrm{C}$ (Fig. 6G,H). The association of TFIIIC was unchanged, but the binding of TFIIIB was reduced in the dst $1-\Delta$ mutant compared with the wild type.

\section{TFIIS affects Pol III transcription in vitro}

To examine the role of TFIIS in Pol III transcription in vitro, we expressed wild-type TFIIS and the TFIIS-E291A mutant form that is unable to stimulate Pol II elongation (Ubukata et al. 2003) as 6xHis fusion proteins in Escherichia coli, and purified the corresponding polypeptides to near homogeneity. We first checked that the wild-type TFIIS was active in stimulating nonspecific Pol II transcription on calf thymus DNA (Sawadogo et al. 1980b), while the TFIIS-E291A protein was not (data not shown). The effect of the wild-type and the E291A mutant TFIIS on Pol III transcription in vitro were examined in a Pol III transcription system reconstituted with all recombinant TFIIIC and TFIIIB and highly purified Pol III (Ducrot et al. 2006). Multiple-round transcription assays were performed with the SUP4 tRNA gene as a template. As observed previously, the SUP4 transcripts generated with TFIIIB recombinant components migrated as two or three diffuse bands on polyacrylamide gels (Fig. 7A, lane 1), which was not the case in the presence of the crude fraction B" (Andrau and Werner 2001). This fraction contains additional factors, like Nhp6 (Braglia et al. 2007), that can restore transcriptional initiation specificity (Kassavetis and Steiner 2006). Western blotting analysis also revealed the presence of TFIIS in the B" fraction /data not shown). Remarkably, adding the purified wild-type TFIIS to the reconstituted transcription system resulted in the formation of the correct length transcript (Fig. 7A, lanes 2-6), in contrast to the purified TFIIS-E291A mutant protein at the same concentration (Fig. 7A, lanes 7-11). The same results were obtained after mixing wildtype and mutant TFIIS preparations, indicating that the mutant TFIIS preparation did not contain interfering components (Fig. 7A, lane 12). Quantification of the transcription signals revealed that the total amount of transcripts did not significantly change in the presence of wild-type or E291A mutant TFIIS. High concentrations of TFIIS $(40 \mathrm{ng} / \mathrm{\mu L})$ started to inhibit the transcription (Fig. 7A, lanes 6-11). In the subsequent assay, we used $5 \mathrm{ng} / \mu \mathrm{L}$ TFIIS.

To demonstrate that TFIIS could contribute to correct start site selection, RNAs generated in a standard multiple-round Pol III transcription assay were purified and analyzed by primer extension. Figure $7 \mathrm{~B}$ showed that, in the absence of TFIIS, transcription initiation occurred at base pairs $+1,+4$, and +8 relative to the start site used in vivo. The addition of $\mathrm{B}^{\prime \prime}$ fraction or of wild-type TFIIS restored a correct start site selection, while the TFIISE291A mutant had no effect. Similar results were obtained on transcripts produced in a single-round assay (Supplemental Fig. S7A). The transcription start sites were analyzed in vivo on SUP4 gene in a nhp6a- $\Delta$ nhp6b- $\Delta$ con- 
Ghavi-Helm et al.
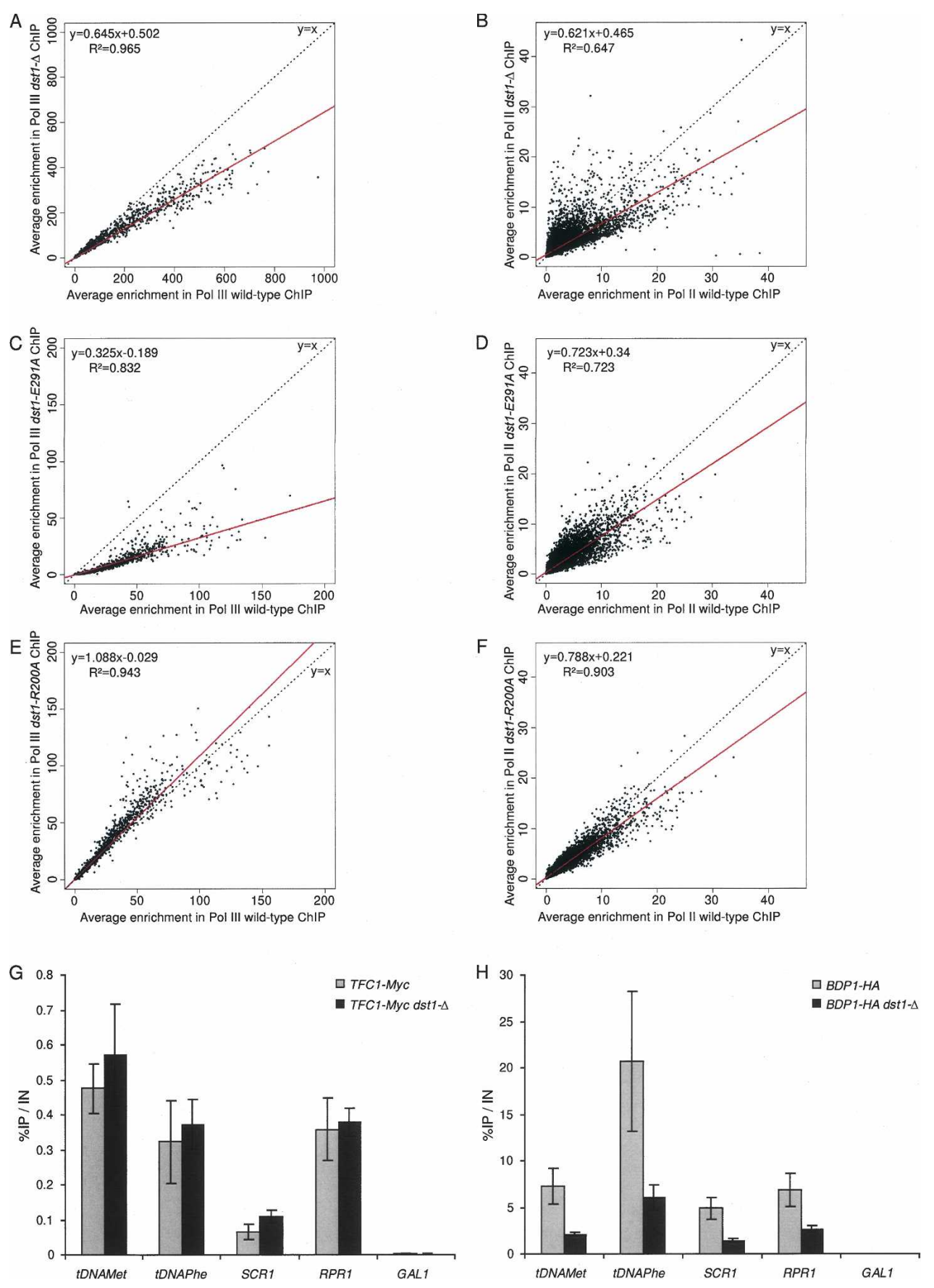

Figure 6. Pol II and Pol III genome-wide enrichment in TFIIS mutants at $16^{\circ} \mathrm{C}$. The genome-wide enrichment of Pol III $(A, C, E)$ or Pol II $(B, D, F)$ in YPD medium $(A, B)$ or SC-leucine medium $(C-F)$ at $16^{\circ} \mathrm{C}$ was assessed from ChIP-chip experiments. A linear regression (red line) and its equation are indicated. The dotted line corresponds to $y=x$. Enrichment of Pol III or Pol II in dst1- $\Delta$ (YGH12) was compared with the wild-type strain (YGH11) $(A, B)$. Enrichment of Pol III or Pol II in dst1-E291A (YGH12/pRS425-dst1E291A) (C,D) and dst1-R200A (YGH12/pRS425-dst1R200A) (E,F) strains was compared with the wild-type strain (YGH12/pRS425-DST1). (G,H) Quantitative ChIP analysis of TFIIIC and TFIIIB enrichment on selected class III genes. dst1- $\Delta$ and wild-type strains were grown in YPD medium at $30^{\circ} \mathrm{C}$ and shifted for $8 \mathrm{~h}$ to $16^{\circ} \mathrm{C}$. Immunoprecipitations were performed using antibodies against 13Myc (9E10) for Tfc1-13Myc and 3HA (12CA5) for Bdp1-3HA. Immunoprecipitated fragments from ChIP experiments were amplified with primers as indicated in the Supplemental Material. The GAL1 ORF was used as a control. Error bars represent the standard deviation between at least three biological replicates.

text and in the presence or absence of DST1 gene. The nhp6 mutant background was used because these proteins were previously implicated in start site selection and could mask the effect of $d s t 1-\Delta$. We could not identify any effect of $d s t 1$ deletion on start site selection in this background (Supplemental Fig. S7B). 
A
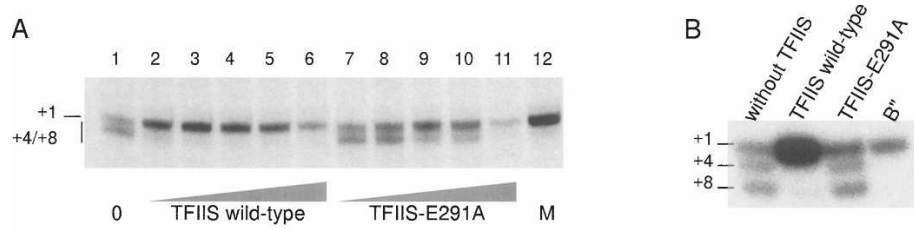
C

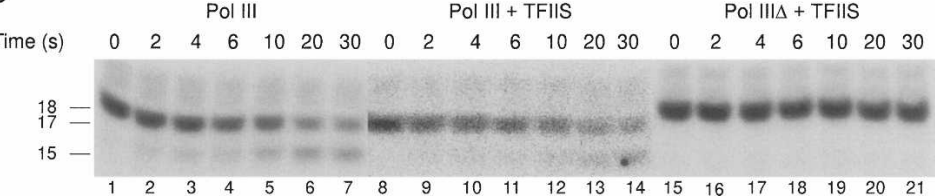

Figure 7. TFIIS stimulates faithful Pol III transcription in vitro. (A) Effect of TFIIS on Pol III transcription. Standard in vitro Pol III transcription on the SUP4 template has been performed as described in the Materials and Methods, in the absence (lane 1) or presence of increasing quantities $(0.1,0.2,0.4,0.8$, and $1.6 \mu \mathrm{g})$ of wild-type TFIIS (lanes 2-6) or mutant TFIIS-E291A (lanes 7-11) or with a mix of wild-type and E291A protein (M, lane 12). Transcription start sites are indicated. $(B)$ Primer extension analysis of start sites used by Pol III on the SUP4 gene in vitro. Transcription reactions were performed in the absence of TFIIS, in the presence of wild-type TFIIS, mutant TFIIS-E291A, or with a B" fraction. Transcription and primer extensions were performed as described in Materials and Methods using a probe hybridizing within the SUP4 intron. Positions of the major transcription start site are indicated. $(C)$ Time-course analysis of RNA cleavage by Pol III. Pol III (lanes 1-14) or Pol III $\Delta$ (lanes 15-21) (Chedin et al. 1998) ternary complexes formed in the presence of 3XTPs were isolated on Sepharose CL-2B as described in the Supplemental Material and then incubated for 10 min with a $50 \mathrm{M}$ excess of purified TFIIS (lanes 8-21) or without TFIIS (lanes 1-7). Ternary complexes were then incubated at $16^{\circ} \mathrm{C}$ in transcription buffer containing 5 $\mathrm{mM} \mathrm{MgCl} 2$ in the absence of nucleotides for various periods of time. The transcript sizes are indicated. Transcription with Pol III $\Delta$ results in the formation of an 18-mer RNA instead of a 17-mer for the wild-type enzyme (Chedin et al. 1998).

To examine whether TFIIS could affect the elongation step of Pol III transcription, the elongation kinetics of Pol III were analyzed on a SUP4 template that can produce a stalled ternary complex after the synthesis of a 17mer transcript in the absence of GTP. The ternary complex was allowed to resume transcription by adding the four NTPs. No significant changes in elongation kinetics were observed in the presence or absence of purified wild-type TFIIS (Supplemental Fig. S8). TFIIS being a cleavage stimulatory factor in Pol II transcription, we wanted to know whether TFIIS could influence the intrinsic cleavage activity of Pol III. Labeled ternary complexes halted by omission of GTP in the transcription reaction were purified and incubated for various periods of time in the presence of $\mathrm{MgCl}_{2}$ to activate the Pol III cleavage activity (Chedin et al. 1998). TFIIS did not stimulate the cleavage activity of wild-type Pol III (Fig. 7C, lanes 1-14, look at the disappearance rate of the 17-mer transcript). Pol III $\Delta$, a RNA polymerase mutant that lacks the Rpc11, Rpc37, and Rpc53 subunits, is not competent for RNA cleavage activity (Chedin et al. 1998; Landrieux et al. 2006). Pol III $\Delta$ was purified from $r p c 37 H A-C t$ mutant as described previously (Landrieux et al. 2006). We observed that the addition of TFIIS did not restore an efficient cleavage by Pol III $\Delta$ (Fig. 7C, lanes 15-21). Taken at face value, in vitro transcription assays suggest a role for TFIIS in start site selection during Pol III transcription.

\section{Discussion}

In this study, we performed a genome-wide location analysis of the TFIIS transcription factor. TFIIS was detected across the whole genome of exponentially growing cells, indicating that the binding of this transcription factor to chromatin is not restricted to NTP-depleted cells. TFIIS and Pol II genome-wide occupancies correlated very well, suggesting that TFIIS is not recruited only when Pol II is stalled. A second and more surprising outcome of our study was that TFIIS could be detected on almost all Pol III-transcribed genes. This result raised the intriguing possibility that TFIIS might operate as a general Pol III-associated factor. We provided substantial in vivo and in vitro data demonstrating that TFIIS is important for Pol III transcription.

Concerning the TFIIS function in Pol II transcription, we observed that TFIIS was associated with a large number of Pol II-transcribed genes in cells growing exponentially in rich medium, in line with previous ChIP analyses of TFIIS on a few class II genes (Prather et al. 2005; Guglielmi et al. 2007). The distribution of TFIIS over distinct Pol II-transcribed regions, including intergenic regions transcribed in short unstable RNAs (Martens et al. 2004; Wyers et al. 2005; Davis and Ares 2006), precisely correlated with Pol II itself, which is consistent with the role of TFIIS as an elongation factor but is not contradictory with an additional role during initiation (Prather et al. 2005; Guglielmi et al. 2007; Kim et al. 2007). In the presence of MPA, the enrichment level of TFIIS genome-wide was only slightly increased compared with that in normal growth condition. Essentially, two models for TFIIS recruitment to Pol II-transcribed genes may be envisioned. TFIIS could be only recruited to arrested Pol II complexes. Alternatively, it might associate and dissociate from the elongating Pol II, independently of transcription arrests, shifting to the cleavage-prone conformation of Pol II if need arises. The latter model would better account for the fact that GTP depletion by MPA, which is likely to promote arrest, does not strongly increase TFIIS occupancy.

The presence of TFIIS at nearly all Pol III-transcribed genes strongly suggests that it is a Pol III transcription factor. This hypothesis is supported by several lines of evidence. (1) TFIIS occupancy correlated well with that of Pol III genome-wide. (2) The occupancy profile of TFIIS closely followed that of Pol III on the SCR1 gene, the longest class III gene. (3) TFIIS coimmunoprecipitated with Pol III after formaldehyde cross-linking. (4) A temperature-sensitive Pol II mutation (rpb1-1) strongly 
reduced TFIIS enrichment at Pol II-transcribed genes but had no effect on its association with Pol III-transcribed genes. Conversely, a temperature-sensitive Pol III mutation (rpc25-S100P) diminished TFIIS binding at Pol IIItranscribed genes with no effect on its association with Pol II-transcribed genes. Thus, the presence of TFIIS on Pol III-transcribed genes depends on Pol III activity and is independent of Pol II. (5) Under low-temperature conditions, the dst1- $\Delta$ mutation affected growth, and diminished Pol III and TFIIIB association with class III genes. (6) The dst1 deletion impaired the translational suppression of ade2-1 (a nonsense UAA mutant) by the SUP4 ${ }^{\text {ochre }}$ suppressor, and reduced the Pol III transcript levels under low-temperature conditions. (7) TFIIS improved Pol III transcription start site selection in vitro. Altogether, these data indicate that TFIIS is a bona fide component of the Pol III transcription machinery. It has been suggested previously that TFIIS could also play a role in Pol I transcription (Sawadogo et al. 1980a; Sawadogo et al. 1981; Schnapp et al. 1996), but a recent report demonstrated that the intrinsic cleavage activity of Pol I requires the Rpa12 subunit, sharing sequence homology with TFIIS (Kuhn et al. 2007). Our ChIP results showed that TFIIS was enriched on the Pol I-transcribed rDNA templates. TFIIS could thus be implicated in Pol I transcription. Interestingly, the Pol III-transcribed 5S rRNA genes, arranged in tandem with the 35S rRNA genes, were not enriched by TFIIS. The reason for the absence of TFIIS on 5S rRNA genes is unknown but could stem from the intragenic binding of TFIIIA factor that is required for $5 \mathrm{~S}$ transcription.

As a Pol II elongation factor, TFIIS strictly depends on the RSADE domain, since inactivating this domain (or deleting the entire TFIIS) makes cells sensitive to NTP-depleting drugs (mycophenolate, 6-azauracil), which is generally seen as a consequence of a defective Pol II-associated cleavage (Exinger and Lacroute 1992; Ubukata et al. 2003). As a factor involved in Pol II initiation, TFIIS is needed for the full recruitment of Pol II to several promoters, especially in the absence of the Med31 subunit of Mediator. This, however, does not depends on the RSADE motif but is impaired in dst1-R200A, a mutation of the TFIIS Pol II-interacting domain (Guglielmi et al. 2007). Turning now to the Pol III-associated role(s) of TFIIS, we found that the RSADE motif is clearly important in this context, since the corresponding mutant form of TFIIS alters start site selection by Pol III in vitro, and since Pol III occupancy is strongly diminished in dst1-E291A cells grown at $16^{\circ} \mathrm{C}$, with a strongly perturbed Pol III transcriptome. The reason why class III genes, the transcription of which is most diminished, differ in dst1- $\Delta$ and $d s t 1-E 291 A$ is presently unknown but might be the consequence of the strong perturbation of Pol III transcription in the latter background. An indirect effect of altered Pol I or Pol II transcription is unlikely because dst1 mutations do not affect 35S rRNA precursor transcription at $16^{\circ} \mathrm{C}$ (data not shown) and because Pol II occupancy defect is not increased in dst1-E291A compared with $d s t 1-\Delta$. In contrast, $d s t 1-R 200 A$ had a limited but significant effect on Pol II occupancy, with no effect at all on Pol III, suggesting that this mutation might specifically affect a Pol II-associated function (Guglielmi et al. 2007).

In the course of this study, we examined the possible implication of TFIIS in the different steps of in vitro Pol III transcription. Omitting TFIIS did not detectably influence Pol III elongation or cleavage activity in vitro but altered start site selection, suggesting that TFIIS might primarily act at the level of Pol III recruitment and/or transcription initiation. This is further supported by our ChIP assays showing that $d s t 1-\Delta$ strongly reduces the presence of Bdp1, the TFIIB-related subunit of the TFIIIB initiation factor of Pol III, with no effect on the TFIIIC initiation factor. In Pol III transcription, start site selection and initiation require the precise targeting of the enzyme by its initiation factors (TFIIIC and TFIIIB) and the opening of the transcription bubble around the start site (Kassavetis and Geiduschek 2006). TFIIS might participate in the initial steps of the Pol III transcription cycle in different ways. TFIIS could bend DNA and facilitate appropriate DNA binding by the transcription machinery as do Nhp6A and Nhp6B (Kassavetis and Steiner 2006). Alternatively, TFIIS could facilitate productive initiation by influencing the interaction of Pol III with basal factors for more accurate enzyme positioning. We favor the second hypothesis since direct binding of TFIIS to DNA has never been shown and since TFIIS and Pol III coimmunoprecipitated in cross-linked extracts.

One could be initially surprised to find TFIIS associated with class III genes, since Pol III has an intrinsic transcript cleavage activity that depends on Rpc11 subunit (Chedin et al. 1998; Alic et al. 2007). Rpc11 has a C-terminal Zn loop that bears an RSADE motif, critical for transcript cleavage, that closely resembles the C-terminal domain of TFIIS (Chedin et al. 1998). Our results suggest that both Rpc11 subunit and TFIIS are required for efficient Pol III transcription, but that their roles are not identical. Recombinant TFIIS (Fig. 7), in contrast to recombinant Rpc11 alone (Chedin et al. 1998), could not restore cleavage activity of Pol III $\Delta$ variant lacking Rpc11, suggesting that TFIIS does not participate in this reaction. Further, we found that Rpc11 and TFIIS bound throughout SCR1 gene as Rpc160 (used as a proxy for Pol III) did. Moreover, TFIIS and Rpc11 coimmunoprecipitated in cross-linked extracts, as did Rpc160, indicating that their binding to class III genes is not mutually exclusive.

In conclusion, there is now mounting evidence that TFIIS controls several levels of DNA transcription. At a subset of Pol II-transcribed gene promoters, it could be recruited and act together with Mediator to recruit Pol II (Prather et al. 2005; Guglielmi et al. 2007; Kim et al. 2007), independently of its transcript cleavage stimulatory activity. TFIIS could also dynamically associate and dissociate from Pol II and stimulate the enzyme intrinsic RNA cleavage activity when needed. In addition, the present study shows that TFIIS is a Pol III transcription factor that stimulates Pol III transcription and may contribute to precise start site selection. 


\section{Materials and methods}

\section{Protein purification}

DB3.1 E. coli cells containing either pDEST17-DST1 or pDEST17dst1E291A were grown at $30^{\circ} \mathrm{C}$ to $0.6 \mathrm{OD}_{600}$. Expression of the $6 \mathrm{xHis}$-TFIIS fusion protein was induced by addition of $1 \mathrm{mM}$ isopropyl-1-thio- $\beta$-D-galactopyranoside. Cells were harvested after $3 \mathrm{~h}$ of induction and resuspended in $20 \mathrm{mM}$ HEPES buffer ( $\mathrm{pH} 7.5$ ) containing $10 \mu \mathrm{M} \mathrm{ZnCl}_{2}, 300 \mathrm{mM} \mathrm{NaCl} 2,10 \%$ glycerol, $10 \mathrm{mM} \beta$-mercaptoethanol, and a set of protease inhibitors (phenyl-methyl-sulfonyl floride, Complete [Roche]). After lysis by sonication at $4^{\circ} \mathrm{C}$, the lysate was clarified by centrifugation in a Beckman JA20 rotor for $25 \mathrm{~min}$ at 12,000 rpm. The 6xHis-TFIIS proteins were purified using an ÄKTA purifier (Amersham Biosciences) on a Hi Trap Chelating HP 5-mL column with a gradient of imidazole from $10 \mathrm{mM}$ to $1 \mathrm{M}$. SDS-PAGE analysis, followed by Coomassie brilliant blue staining, showed that the fusion proteins were purified to near homogeneity.

\section{ChIP and genome-wide ChIP-chip}

Cross-linked chromatin was prepared essentially as described previously (Kuras and Struhl 1999; Kuras et al. 2003). Cells were grown exponentially to $0.6 \mathrm{OD}_{600}$ and cross-linked with $1 \%$ formaldehyde for $10 \mathrm{~min}$. The 3HA- and $13 \mathrm{Myc}$-tagged proteins were immunoprecipitated with 12CA5 and 9E10 antibodies, respectively; Pol II was immunoprecipitated with 8WG16 antiCTD antibody (Covance), and bound to IgG magnetic beads (Dynabead). Immune complexes were washed as described previously (Kuras and Struhl 1999). Cross-link reversal and DNA purification were performed as described (Kuras et al. 2003), except that the final elution was in $50 \mu \mathrm{L}$. Immunoprecipitated DNA was analyzed by quantitative real-time PCR on an ABI Prism 7000 or 7300 machine (Applied Biosystems). The PCR reactions were carried out in $25 \mu \mathrm{L}$ containing $0.4 \mu \mathrm{M}$ each primer, and $12.5 \mu \mathrm{L}$ of mastermix SYBR green PCR reaction (Applied Biosystems). Relative quantification using a standard curve method was performed and the occupancy level for a specific fragment was defined as the ratio of immunoprecipitated DNA over total DNA. GAL1 ORF region was used as a nontranscribed control.

Ligation-mediated PCR was done as described previously (Ren et al. 2000), except that amino-allyl conjugated dUTP $(150 \mu \mathrm{M}$ final) was used and only 30 cycles of PCR were performed. The PCR products were purified using a Microcon YM-30 filter. Amino-allyl modified DNA was recovered with $20 \mu \mathrm{L}$ of $\mathrm{H}_{2} \mathrm{O}$ and the DNA was lyophilized. DNA was labeled as previously described (Harismendy et al. 2003), except that unincorporated dyes were removed using a QIAquick PCR Purification Kit (Qiagen). Labeled DNA was recovered with $100 \mu \mathrm{L}$ of buffer EB, and ethanol precipitated. Hybridization and washing conditions were as described previously (Lee et al. 2006). Microarrays were obtained from Agilent Technologies and feature 41,418 (G4486A) or 41,776 (G4493A) 60-mer oligonucleotide probe spots, with an average density of one probe each $266 \mathrm{bp}$ of the yeast genome. Images of $\mathrm{Cy} 5$ and $\mathrm{Cy} 3$ fluorescence intensities were generated using the Genepix 4000B scanner (Molecular Devices) and extracted using GenePix Pro 6 software (Molecular Devices). At least two biological replicates were performed for each experiment.

\section{Data analysis}

The computational data analysis was performed in $\mathrm{R}$ using the limma package (Smyth et al. 2005) from the bioconductor project (http://www.bioconductor.org). After subtraction of the local background, the data from both channels were median normalized, and log ratios of signal intensities were generated for each feature. The log ratios were processed by fitting a linear model for each feature in order to calculate the average log ratio between replicates. $P$-values were then calculated by performing an empirical Bayes moderated $t$-statistic test, and adjusted for multiple testing by Benjamini and Hochberg false discovery rate (FDR) method. The complete raw data set is available at Array Express (http://www.ebi.ac.uk/arrayexpress) under accession number E-MTAB-10. Visualization of ChIP-enriched genomic regions was performed using an adaptation of the Ringo package (Toedling et al. 2007). GO analysis was performed using GOstats package.

\section{Pol III transcriptome analysis}

Microarray hybridization was performed as described previously (Ciesla et al. 2007). Briefly, $20 \mu \mathrm{g}$ of total RNA was reverse transcribed with specific primers designed to hybridize to the $3^{\prime}$ end of all mature tRNAs. cDNA was labeled with Cy3 and Cy5 dyes (Amersham) and hybridized on a Pol III-specific microarray (O. Harismendy, pers. comm.) harboring all the different tRNA genes, as well as all the other genes transcribed by Pol III.

\section{In vitro transcription and primer extension assays}

Standard Pol III in vitro transcription reactions on the SUP4 template were performed as described previously (Ducrot et al. 2006; Alic et al. 2007) in $40 \mu \mathrm{L}$ of transcription buffer. pRS316SUP4 plasmid (150 ng) was incubated for $20 \mathrm{~min}$ at $25^{\circ} \mathrm{C}$ in the presence of $100 \mathrm{ng}$ of $\mathrm{rTFIIIC}, 20 \mathrm{ng}$ of $\mathrm{rTBP}, 10 \mathrm{ng}$ of $\mathrm{rBdp} 1,10$ ng of rBrf1, $100 \mathrm{ng}$ of highly purified Pol III, and $0.5 \mu \mathrm{g}$ of purified $\mathrm{B}^{\prime \prime}$ fraction or different concentrations of TFIIS protein (wild-type or E291A mutant). Transcription was started by the addition of $600 \mu \mathrm{M}$ A/C/GTP, $300 \mu \mathrm{M}$ UTP, $25 \mu \mathrm{M}\left[\alpha-{ }^{32} \mathrm{P}\right] \mathrm{UTP}$ (400 Ci/mmol), and allowed to proceed for $15 \mathrm{~min}$.

Primer extension assays were performed as described previously (Andrau and Werner 2001) on the SUP4 template with 200 $\mu \mathrm{M}$ unlabeled ATP, CTP, UTP, and GTP. Single-round assays were performed as described, except that $125 \mu \mathrm{M}$ unlabeled ATP, CTP, UTP, GTP, and $0.3 \mathrm{mg} / \mathrm{mL}$ heparin were used (Kassavetis and Steiner 2006).

The reaction products were analyzed by electrophoresis on denaturing polyacrylamide gels $(7 \%$ for in vitro transcription and primer extension assay). Gels were autoradiographed using MR film with an intensifying screen (Kodak). Quantifications were performed with Image Quant software (Molecular Dynamics).

\section{Acknowledgments}

We thank M. Wery, D. Després, E. Shematorova, M. Boguta, K. Struhl, D. Stillman, and O. Gadal for yeast strains or plasmids; C. Kane for anti-TFIIS antibody; the SPI (CEA/Saclay) for monoclonal antibodies; C. Conesa, O. Harismendy, and S.G.F. (CEA/ Evry) for Pol III transcriptome arrays and protocols; M. Riva for the yeast Pol II preparation and advice on Pol II transcription assays; C. Carles, N. Ayoub, and N. Alic for the yeast Pol III $\Delta$ preparation and advice in cleavage assays; and C. Ducrot for Pol III transcription factor preparations. We also thank O. Lefebvre and A. Briand for useful advice, and A. Sentenac and C. Mann for critical reading of the manuscript. Y.G. and M.M. were supported by a "Contrat de Formation par la Recherche" from the CEA.

\section{References}

Alic, N., Ayoub, N., Landrieux, E., Favry, E., Baudouin-Cornu, P., Riva, M., and Carles, C. 2007. Selectivity and proofread- 
ing both contribute significantly to the fidelity of RNA polymerase III transcription. Proc. Nat1. Acad. Sci. 104: 1040010405.

Andrau, J.C. and Werner, M. 2001. B"-associated factor(s) involved in RNA polymerase III preinitiation complex formation and start-site selection. Eur. J. Biochem. 268: 5167-5175.

Awrey, D.E., Weilbaecher, R.G., Hemming, S.A., Orlicky, S.M., Kane, C.M., and Edwards, A.M. 1997. Transcription elongation through DNA arrest sites. A multistep process involving both RNA polymerase II subunit RPB9 and TFIIS. J. Biol. Chem. 272: 14747-14754.

Awrey, D.E., Shimasaki, N., Koth, C., Weilbaecher, R., Olmsted, V., Kazanis, S., Shan, X., Arellano, J., Arrowsmith, C.H., Kane, C.M., et al. 1998. Yeast transcript elongation factor (TFIIS), structure and function. II: RNA polymerase binding, transcript cleavage, and read-through. J. Biol. Chem. 273: 22595-22605.

Borukhov, S., Sagitov, V., and Goldfarb, A. 1993. Transcript cleavage factors from E. coli. Cell 72: 459-466.

Braglia, P., Dugas, S.L., Donze, D., and Dieci, G. 2007. Requirement of Nhp6 proteins for transcription of a subset of tRNA genes and heterochromatin barrier function in Saccharomyces cerevisiae. Mol. Cell. Biol. 27: 1545-1557.

Chedin, S., Riva, M., Schultz, P., Sentenac, A., and Carles, C. 1998. The RNA cleavage activity of RNA polymerase III is mediated by an essential TFIIS-like subunit and is important for transcription termination. Genes \& Dev. 12: 3857-3871.

Chen, W., Tabor, S., and Struhl, K. 1987. Distinguishing between mechanisms of eukaryotic transcriptional activation with bacteriophage T7 RNA polymerase. Cell 50: 1047-1055.

Ciesla, M., Towpik, J., Graczyk, D., Oficjalska-Pham, D., Harismendy, O., Suleau, A., Balicki, K., Conesa, C., Lefebvre, O., and Boguta, M. 2007. Maf1 is involved in coupling carbon metabolism to RNA Polymerase III transcription. Mol. Cell. Biol. 27: 7693-7702.

Davis, C.A. and Ares Jr., M. 2006. Accumulation of unstable promoter-associated transcripts upon loss of the nuclear exosome subunit Rrp6p in Saccharomyces cerevisiae. Proc. Nat1. Acad. Sci. 103: 3262-3267.

Ducrot, C., Lefebvre, O., Landrieux, E., Guirouilh-Barbat, J., Sentenac, A., and Acker, J. 2006. Reconstitution of the yeast RNA polymerase III transcription system with all recombinant factors. J. Biol. Chem. 281: 11685-11692.

Exinger, F. and Lacroute, F. 1992. 6-Azauracil inhibition of GTP biosynthesis in Saccharomyces cerevisiae. Curr. Genet. 22: 9-11.

Fish, R.N. and Kane, C.M. 2002. Promoting elongation with transcript cleavage stimulatory factors. Biochim. Biophys. Acta 1577: 287-307.

Geiduschek, E.P. and Kassavetis, G.A. 2001. The RNA polymerase III transcription apparatus. J. Mol. Biol. 310: 1-26.

Guglielmi, B., Soutourina, J., Esnault, C., and Werner, M. 2007. TFIIS elongation factor and Mediator act in conjunction during transcription initiation in vivo. Proc. Natl. Acad. Sci. 104: 16062-16067.

Harismendy, O., Gendrel, C.G., Soularue, P., Gidrol, X., Sentenac, A., Werner, M., and Lefebvre, O. 2003. Genome-wide location of yeast RNA polymerase III transcription machinery. $E M B O$ I. 22: 4738-4747.

Hausner, W., Lange, U., and Musfeldt, M. 2000. Transcription factor $S$, a cleavage induction factor of the archaeal RNA polymerase. J. Biol. Chem. 275: 12393-12399.

Kassavetis, G.A. and Geiduschek, E.P. 2006. Transcription factor TFIIIB and transcription by RNA polymerase III. Biochem. Soc. Trans. 34: 1082-1087.

Kassavetis, G.A. and Steiner, D.F. 2006. Nhp6 is a transcrip- tional initiation fidelity factor for RNA polymerase III transcription in vitro and in vivo. J. Biol. Chem. 281: 7445-7451.

Kettenberger, H., Armache, K.J., and Cramer, P. 2003. Architecture of the RNA polymerase II-TFIIS complex and implications for mRNA cleavage. Cell 114: 347-357.

Kim, B., Nesvizhskii, A.I., Rani, P.G., Hahn, S., Aebersold, R., and Ranish, J.A. 2007. The transcription elongation factor TFIIS is a component of RNA polymerase II preinitiation complexes. Proc. Nat1. Acad. Sci. 104: 16068-16073.

Kuhn, C.D., Geiger, S.R., Baumli, S., Gartmann, M., Gerber, J., Jennebach, S., Mielke, T., Tschochner, H., Beckmann, R., and Cramer, P. 2007. Functional architecture of RNA polymerase I. Cell 131: 1260-1272.

Kulish, D. and Struhl, K. 2001. TFIIS enhances transcriptional elongation through an artificial arrest site in vivo. Mol. Cell. Biol. 21: 4162-4168.

Kuras, L. and Struhl, K. 1999. Binding of TBP to promoters in vivo is stimulated by activators and requires Pol II holoenzyme. Nature 399: 609-613.

Kuras, L., Borggrefe, T., and Kornberg, R.D. 2003. Association of the Mediator complex with enhancers of active genes. Proc. Natl. Acad. Sci. 100: 13887-13891.

Landrieux, E., Alic, N., Ducrot, C., Acker, J., Riva, M., and Carles, C. 2006. A subcomplex of RNA polymerase III subunits involved in transcription termination and reinitiation. EMBO J. 25: $118-128$.

Lee, T.I., Johnstone, S.E., and Young, R.A. 2006. Chromatin immunoprecipitation and microarray-based analysis of protein location. Nat. Protoc. 1: 729-748.

Malagon, F., Tong, A.H., Shafer, B.K., and Strathern, J.N. 2004. Genetic interactions of DST1 in Saccharomyces cerevisiae suggest a role of TFIIS in the initiation-elongation transition. Genetics 166: 1215-1227.

Martens, J.A., Laprade, L., and Winston, F. 2004. Intergenic transcription is required to repress the Saccharomyces cerevisiae SER3 gene. Nature 429: 571-574.

Mason, P.B. and Struhl, K. 2005. Distinction and relationship between elongation rate and processivity of RNA polymerase II in vivo. Mol. Cell 17: 831-840.

Moqtaderi, Z. and Struhl, K. 2004. Genome-wide occupancy profile of the RNA polymerase III machinery in Saccharomyces cerevisiae reveals loci with incomplete transcription complexes. Mol. Cell. Biol. 24: 4118-4127.

Nakanishi, T., Shimoaraiso, M., Kubo, T., and Natori, S. 1995. Structure-function relationship of yeast S-II in terms of stimulation of RNA polymerase II, arrest relief, and suppression of 6-azauracil sensitivity. J. Biol. Chem. 270: 8991-8995.

Natori, S., Takeuchi, K., Takahashi, K., and Mizuno, D. 1973. DNA dependent RNA polymerase from Ehrlich ascites tumor cells. II. Factors stimulating the activity of RNA polymerase II. J. Biochem. 73: 879-888.

Nonet, M., Scafe, C., Sexton, J., and Young, R. 1987. Eucaryotic RNA polymerase conditional mutant that rapidly ceases mRNA synthesis. Mol. Cell. Biol. 7: 1602-1611.

Olmsted, V.K., Awrey, D.E., Koth, C., Shan, X., Morin, P.E., Kazanis, S., Edwards, A.M., and Arrowsmith, C.H. 1998. Yeast transcript elongation factor (TFIIS), structure and function. I: NMR structural analysis of the minimal transcriptionally active region. J. Biol. Chem. 273: 22589-22594.

Opalka, N., Chlenov, M., Chacon, P., Rice, W.J., Wriggers, W., and Darst, S.A. 2003. Structure and function of the transcription elongation factor GreB bound to bacterial RNA polymerase. Cell 114: 335-345.

Pokholok, D.K., Hannett, N.M., and Young, R.A. 2002. Exchange of RNA polymerase II initiation and elongation factors during gene expression in vivo. Mol. Cell 9: 799-809. 
Prather, D.M., Larschan, E., and Winston, F. 2005. Evidence that the elongation factor TFIIS plays a role in transcription initiation at GAL1 in Saccharomyces cerevisiae. Mol. Cell. Biol. 25: 2650-2659.

Ren, B., Robert, F., Wyrick, J.J., Aparicio, O., Jennings, E.G., Simon, I., Zeitlinger, J., Schreiber, J., Hannett, N., Kanin, E., et al. 2000. Genome-wide location and function of DNA binding proteins. Science 290: 2306-2309.

Roberts, D.N., Stewart, A.J., Huff, J.T., and Cairns, B.R. 2003. The RNA polymerase III transcriptome revealed by genomewide localization and activity-occupancy relationships. Proc. Natl. Acad. Sci. 100: 14695-14700.

Roberts, D.N., Wilson, B., Huff, J.T., Stewart, A.J., and Cairns, B.R. 2006. Dephosphorylation and genome-wide association of Mafl with Pol III-transcribed genes during repression. Mol. Cell 22: 633-644.

Sawadogo, M., Huet, J., and Fromageot, P. 1980a. Similar binding site for P37 factor on yeast RNA polymerases A and B. Biochem. Biophys. Res. Commun. 96: 258-264.

Sawadogo, M., Sentenac, A., and Fromageot, P. 1980b. Interaction of a new polypeptide with yeast RNA polymerase B. $J$. Biol. Chem. 255: 12-15.

Sawadogo, M., Lescure, B., Sentenac, A., and Fromageot, P. 1981. Native deoxyribonucleic acid transcription by yeast RNA polymerase-P37 complex. Biochemistry 20: 3542-3547.

Schnapp, G., Graveley, B.R., and Grummt, I. 1996. TFIIS binds to mouse RNA polymerase I and stimulates transcript elongation and hydrolytic cleavage of nascent rRNA. Mol. Gen. Genet. 252: 412-419.

Shilatifard, A., Conaway, R.C., and Conaway, J.W. 2003. The RNA polymerase II elongation complex. Annu. Rev. Biochem. 72: 693-715.

Smyth, G.K., Michaud, J., and Scott, H.S. 2005. Use of withinarray replicate spots for assessing differential expression in microarray experiments. Bioinformatics 21: 2067-2075.

Toedling, J., Sklyar, O., and Huber, W. 2007. Ringo-An R/Bioconductor package for analyzing ChIP-chip readouts. BMC Bioinformatics 8: 221; doi: 10.1186/1471-2105-8-221.

Ubukata, T., Shimizu, T., Adachi, N., Sekimizu, K., and Nakanishi, T. 2003. Cleavage, but not read-through, stimulation activity is responsible for three biologic functions of transcription elongation factor S-II. J. Biol. Chem. 278: 85808585.

Wery, M., Shematorova, E., Van Driessche, B., Vandenhaute, J., Thuriaux, P., and Van Mullem, V. 2004. Members of the SAGA and Mediator complexes are partners of the transcription elongation factor TFIIS. EMBO T. 23: 4232-4242.

Wyers, F., Rougemaille, M., Badis, G., Rousselle, J.C., Dufour, M.E., Boulay, J., Regnault, B., Devaux, F., Namane, A., Seraphin, B., et al. 2005. Cryptic pol II transcripts are degraded by a nuclear quality control pathway involving a new poly(A) polymerase. Cell 121: 725-737.

Zaros, C. and Thuriaux, P. 2005. Rpc25, a conserved RNA polymerase III subunit, is critical for transcription initiation. Mol. Microbiol. 55: 104-114. 


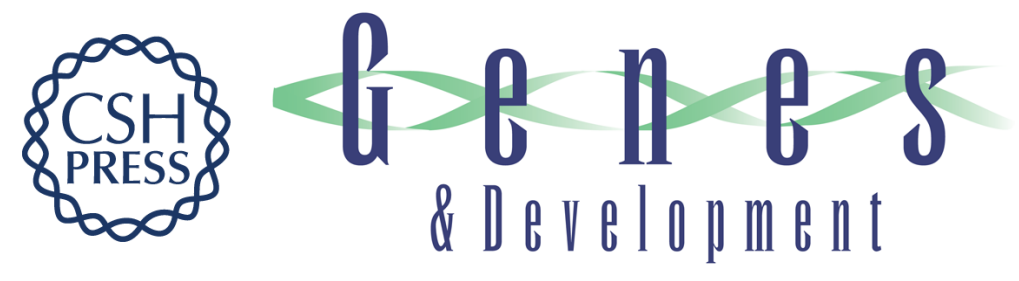

\section{Genome-wide location analysis reveals a role of TFIIS in RNA polymerase III transcription}

Yad Ghavi-Helm, Magali Michaut, Joël Acker, et al.

Genes Dev. 2008, 22:

Access the most recent version at doi:10.1101/gad.471908

Supplemental http://genesdev.cshlp.org/content/suppl/2008/07/01/22.14.1934.DC1
Material

References This article cites 52 articles, 25 of which can be accessed free at:

http://genesdev.cshlp.org/content/22/14/1934.full.html\#ref-list-1

License

Email Alerting

Receive free email alerts when new articles cite this article - sign up in the box at the top

Service

right corner of the article or click here.

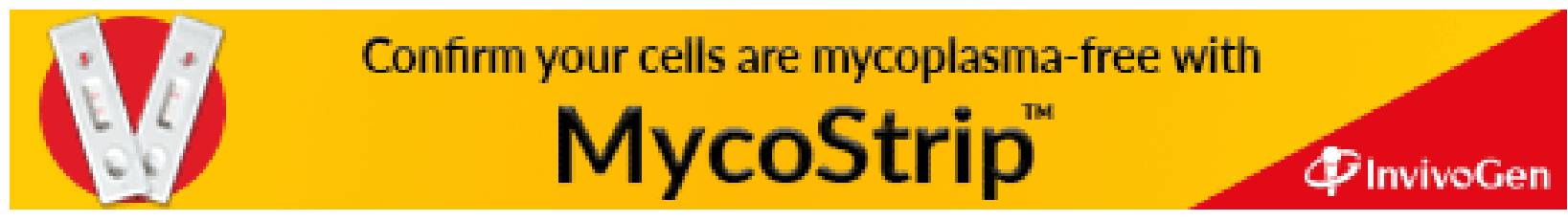

